\title{
A blind channel estimation under inter-carrier interference in QAM-OFDM
}

\author{
Vithaya SAYVISITH, Tetsuya KIMURA, Fuminari NAKAGAWA, \\ Hiroshi SHIRATSUCHI, Naomi HARATANI, Hiromu GOTANDA \\ Graduate School of Advanced Technology, Kinki University, \\ 11-6 Kayanomori, Iizuka-shi, 820-8555 Japan
}

E-mail: \{sira, haratani, gotanda\}@fuk.kindai.ac.jp

\begin{abstract}
This paper proposes a blind estimation of the fading coefficients without using the pilot symbols in QAMOFDM systems subjected to carrier frequency offset (CFO) where the phases of the fading coefficients are within $\pm \pi / 4$. First, under a frequency selective fading channel, the inter-carrier interference (ICI) due to CFO is formulated as an ICA model, the permutation and scale uncertainty inherent in ICA is resolved and CFO is estimated. Next, based on the CFO estimation results, the fading coefficients are estimated by use of the fact that symbol constellation at each sub-carrier is stretched/shrunk and rotated according to the value of its fading coefficient. Then, the unknown transmitted symbols are restored by use of the estimated coefficients. Finally, the validity of the proposed approach is confirmed from several simulations for 16QAM-OFDM.
\end{abstract}

\section{Introduction}

The orthogonal frequency division multiplex (OFDM) system has been adopted for wireless LAN and Digital Terrestrial Television, and it is expected for next generation mobile radio communication [1]. It is, however, very sensitive to carrier frequency offset (CFO) which results in inter-carrier interference (ICI) between the sub-carriers and deteriorates the system performance.

Some practical CFO estimation methods have been proposed using the pilot symbols [2] or the guard interval [3][4] . In addition to these practical CFO estimations, blind $\mathrm{CFO}$ estimations have been also proposed using null sub-carrier [5] or over sampling procedure [6]. Although these methods can be considered as blind methods, they assume the usage of redundant sub-carriers or additional sampling mechanism. Besides they can not estimate the communication channels.

Based on independent component analysis (ICA), we have proposed a blind CFO estimation by using only received symbols suffered from ICI under the AWGN channel [7]: The ICI problem due to CFO is formulated in the ICA framework and decomposed symbols is generated from the separated symbol resulted from the natural gradient (NG) algorithm. Next, the permu- tation and scale uncertainty inherent in ICA is resolved by use of the fact that the decomposed symbols inherit the unknown interference characteristics, i.e., ICI due to $\mathrm{CFO}$. Then, CFO is estimated from the ratio of the decomposed symbols, and the transmitted symbols are restored. At the same time, computational reduction of NG algorithm is attempted taking advantage of the fact that each element in the mixing matrix is not independent but dependent on the value of CFO.

In the present paper, the above method [7] is extended to QAM-OFDM systems subjected to not only CFO but also frequency selective fading of which the phases are within $\pm \pi / 4$. First, under the frequency selective fading channel, the inter-carrier interference due to $\mathrm{CFO}$ is formulated as an ICA model, the permutation and scale uncertainty inherent in ICA is resolved and $\mathrm{CFO}$ is estimated. Next, based on the CFO estimation results, the fading coefficients are estimated by use of the fact that symbol constellation at each sub-carrier is stretched/shrunk and rotated according to the value of the fading coefficient. Then, the unknown transmitted symbols are restored using the estimated coefficients. Finally, the validity of the proposed approach is confirmed from several simulations for 16QAM-OFDM systems.

\section{CFO estimation by ICA}

\subsection{CFO problem under fading channel}

In QAM-OFDM systems, the $m(=0,1, \cdots, M-$ $1)$-th transmitted symbol block is denoted by $s(m)$ $=\left[s_{0}(m), s_{1}(m), \cdots, s_{N-1}(m)\right]^{T}$, and its corresponding received symbol block is denoted by $x(m)=$ $\left[x_{0}(m), x_{1}(m), \cdots, x_{N-1}(m)\right]^{T}$, where $T$ denotes transposition, $M$ and $N$ are the numbers of blocks and subcarriers, respectively. The received symbol is equal to the transmitted symbol in the $i$-th sub-carrier as $x_{i}(m)=s_{i}(m)$ when there exists no channel distortion. However, the received symbol through a fading channel is given as

$$
x_{i}(m)=h_{i} s_{i}(m)
$$


where $h_{i}$ is an unknown complex number called a fading coefficient in the $i$-th sub-carrier ${ }^{1}$. Under the environment there exist CFO in addition to the fading, the received symbol in the $l$-th sub-carrier is given by

$$
x_{l}(m)=\sum_{i=0}^{N-1} \alpha_{l i} h_{i} s_{i}(m)
$$

because of ICI from the other sub-carriers where $\alpha_{l i}$ is an interference intensity defined by

$$
\alpha_{l i}=\frac{\sin (\pi(i-l+\epsilon))}{N \sin (\pi(i-l+\epsilon) \cdot / N)} e^{\frac{j \pi(i-l+\epsilon)(N-1)}{N}}
$$

and is related as $\alpha_{l i}=\alpha_{i-l}$. The frequency offset $\Delta f$ is normalized as $\epsilon=N \Delta f T_{s}$ where $T_{s}$ is the symbol time. For the sake of convenience, we call $\epsilon$ as CFO, and the block number $m$ is omitted except for the case in which it is necessary.

\subsection{ICA formulation of ICI problem}

Under the assumption that $N$ transmitted symbols $\left\{s_{i} \mid i=0,1, \cdots, N-1\right\}$ are statistically independent, the received symbol $x=\left[x_{0}, x_{1}, \cdots, x_{N-1}\right]^{T}$ is formulated in the ICA framework as follows:

$$
x=A s^{\prime}
$$

where $A=\left[\alpha_{l i}\right]$ is an unknown interference (mixing) matrix, $s^{\prime}=\left[s_{0}^{\prime}, s_{1}^{\prime}, \cdots, s_{N-1}^{\prime}\right]^{T}$ is the unknown transmitted symbol distorted by the fading and its elements are expressed in terms of the fading coeffiecient $h_{i}$ as $s_{i}^{\prime}=h_{i} s_{i}$. For the sake of convenience, we call $s_{i}^{\prime}$ a provisional symbol.

In ICA framework, the uninterfering (demixing) matrix $W$ is updated by NG algorithm [9][10] as follows:

$$
W=W-\eta\left[\phi(u) u^{H}-I\right] W
$$

where $\eta$ is an update coefficient, $\phi(\cdot)$ is a nonlinear function, $I$ is a unit matrix and $H$ denotes conjugate transposition. The separated symbol $u=\left[u_{\tilde{0}}, u_{\overline{1}}, \cdots, u_{\widetilde{N-1}}\right]^{T}$ is calculated as

$$
u=W x
$$

Note that the subscript of $u_{\bar{i}}$ is denoted as $\tilde{i}$ in order to refer to permutation and scale uncertainty, i.e., the order of the $i$-th element of $u$ is not necessarily coincident to that of the $\tilde{i}$-th element of $s^{\prime}$ and the scale of the former is different from that of the latter even under no permutation.

In terms of $u_{\tilde{i}}$, a decomposed symbol $\xi_{\tilde{i}}=$ $\left[\xi_{0 \bar{i}}, \xi_{1 \tilde{i}}, \cdots, \xi_{l \bar{i}}, \cdots, \xi_{N-1, \tilde{i}}\right]^{T}$ is defined as follows[11]:

$$
\xi_{\bar{i}}=W^{-1}\left[0, \cdots, 0, u_{\tilde{i}}, 0, \cdots, 0\right]^{T}
$$

The mechanism generating these decomposed symbols is identical to the interfering mechanism of ICI. See [7]

\footnotetext{
${ }^{1}$ See [8] for the detailed derivation where the guard interval is involved.
}

[12] for the proof and the details. Using this useful property, we can search the number $\hat{i}$ among $N$ candidates $(l=0, \cdots, N-1)$ such that the absolute value of $\xi_{l i}$ should be maximum as

$$
\hat{i}=\arg \max _{l}\left|\xi_{l \tilde{i}}\right|
$$

From the search result, we can determine the order of transmitted symbol corresponding to the $\tilde{i}$-th decomposed symbol $\xi_{\tilde{i}}$ as $\tilde{i}=\hat{i}$. In other words, the decomposed symbol $\xi_{\bar{i}}$ can be specified to correspond to the transmitted symbol $s_{\hat{i}}$. Carrying out this procedure over all the $\xi_{\tilde{i}}(\tilde{i}=\tilde{0}, \tilde{1}, \cdots, \widetilde{N-1})$, we can obtain the decomposed symbol without permutation and scale uncertainty [7]. Rewriting the decomposed symbol specified in this manner as $\hat{\xi}_{i}$, it can be expressed in terms of the provisional symbol as follows:

$$
\hat{\xi}_{l i}=\alpha_{l i} s_{i}^{\prime} \quad(l=0,1, \cdots, N-1)
$$

\subsection{CFO estimation}

Let's denote the ratio of the $l$-th and the $i$-th elements of $\hat{\xi}_{i}$ as $r_{l i}$. Then it is found from Eq. (9) that the ratio finally becomes equivalent to that of the interference intensities as follows:

$$
r_{l i}=\hat{\xi}_{l i} / \hat{\xi}_{i i}=\alpha_{l i} s_{i}^{\prime} / \alpha_{i i} s_{i}^{\prime}=\alpha_{l i} / \alpha_{i i} \quad(l \neq i)
$$

We call $r_{l i}$ a decomposed symbol ratio. Substitution of Eq. (3) into Eq. (10) leads to the following relation.

$r_{l i}=e^{\frac{-j \pi(i-l)}{N}}\left\{\cos \frac{\pi(i-l)}{N}+\sin \frac{\pi(i-l)}{N} \cot \frac{\pi \epsilon}{N}\right\}^{-1}$

From this equation, we can get candidates for the CFO estimate as

$$
\epsilon_{l i}=\frac{N}{\pi} \cot ^{-1}\left\{\frac{e^{-j \pi(i-l) / N}}{r_{l i} \sin \pi(i-l) / N}-\cot \frac{\pi(i-l)}{N}\right\}
$$

The first term in $\cot ^{-1}\{\cdot\}$ in the right-hand side of Eq. (12) must be theoretically a real number, but $\hat{r}_{l i}$ given numerically is a complex number. . It is also derived from Eq. (10) that $\left|r_{l i}\right|$ becomes the maximum when $l=i-1$.

Considering the above facts, we calculate the decomposed symbol ratio as

$$
\hat{r}=\frac{1}{N-1} \sum_{i=1}^{N-1} \operatorname{Re}\left\{\hat{r}_{i-1, i}\right\}
$$

and then estimate $\mathrm{CFO}[7]$ as

$$
\hat{\epsilon}=\frac{N}{\pi} \cot ^{-1}\left\{\frac{e^{-j \pi / N}}{\hat{r} \sin \pi / N}-\cot \frac{\pi}{N}\right\}
$$

where $\operatorname{Re}\{\cdot\}$ denotes the real part of the complex number. 


\subsection{NG algorithm with a constraint}

The ICA algorithm is originally formulated on the assumption that the elements of the unknown mixing matrix are independent. Then the amount of computation achieving the convergence is very large in general. However, the elements $\alpha_{l i}$ of the mixing matrix $A$ in Eq. (4) are not independent but dependent on a single variable $\epsilon$ as shown in Eq. (3). From the view point of reducing the computational burden, we have developed a new algorithm which incorporates Eq. (3) into the NG algorithm as a constraint. See [7] for all the details.

\section{Channel and symbol estimation}

Under QAM-OFDM systems, an estimation of $h_{i}$ is proposed where the phase of the fading coefficient $h_{i}$ is restricted within $\pm \pi / 4$ at all the sub-carriers. By use of the estimation results, the transmitted symbols are restored.

\subsection{Restoration of provisional symbols}

Denote the estimate of the interference matrix $A$ as $\hat{A}$, of which elements are given as $\hat{\alpha}_{l i}\left(=\hat{\alpha}_{i-l}\right)$ by substituting the CFO estimate $\hat{\epsilon}$ of Eq. (14) into Eq. (3). The provisional symbol $s^{\prime}$ without ICI is then restored from the received symbol $x$ as follows:

$$
\hat{s}^{\prime}(m)=\hat{A}^{-1} \boldsymbol{x}(m)
$$

where $m(=0,1, \cdots, M-1)$ is the block number.

\subsection{Fading coefficient estimation}

The relation $s_{i}^{\prime}=h_{i} s_{i}$ is expressed in the polar coordinates as follows.

$$
\left|s_{i}^{\prime}\right| e^{j L s_{i}^{\prime}}=\left|h_{i}\right|\left|s_{i}\right| e^{j\left(L h_{i}+L s_{i}\right)}
$$

It is then found that the provisional symbol $s_{i}^{\prime}$ is the complex number to be stretched/shrunk and rotated from the transmitted symbol $s_{i}$ by $\left|h_{i}\right|$ and $L h_{i}$, respectively. Therefore, if the absolute values and phases of $s_{i}$ and $s_{i}^{\prime}$ are known, the absolute value $\left|h_{i}\right|$ and the phase $\angle h_{i}$ of $h_{i}$ can be calculated as follows:

$$
\left|h_{i}\right|={ }_{1}\left|s_{i}^{\prime}\right| /\left|s_{i}\right| \quad \angle h_{i^{\prime}}=\angle s_{i}^{\prime}-\angle s_{i}
$$

In the following, $\left|h_{i}\right|$ and $\angle h_{i}$ are estimated by comparison of two constellations of $\left\{s_{i}(m)\right\}$ and $\left\{s_{i}^{\prime}(m)\right\}$ where $0 \leq m \leq M-1$. The constellation of the transmitted symbols $\left\{s_{i}(m)\right\}$ is automatically determined depending on the modulation scheme prescribed by the protocol between the transmitter and the receiver. In the case of QAM modulation, the constellation of the transmitted symbols $\left\{s_{i}\right\}$ is expressed by the four points with the mark "O" as shown in Fig. 1. The absolute value and the phase of these four points are given respectively as $\left|s_{i}\right|(=\sqrt{2})$ and $\angle s_{i}(= \pm \pi / 4, \pm 3 \pi / 4)$ and they can be dealt with as known.

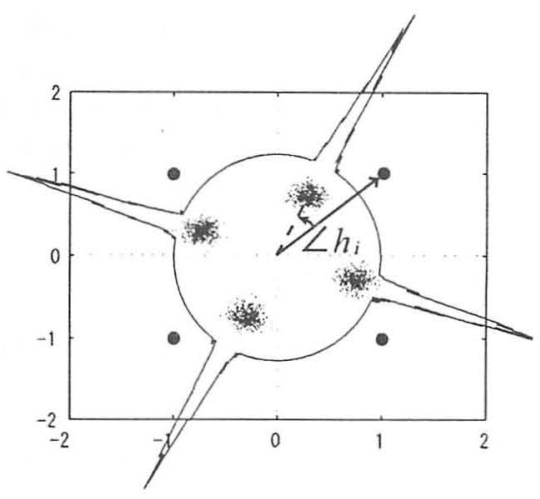

Fig. 1: Constellation of $s_{i}$ and $\hat{s}_{i}^{\prime}$ (4QAM).

On the other hand, the constellation of the restored provisional symbols $\left\{\hat{s}^{\prime}(m)\right\}$ is distributed in four clusters because of the additive noise channel as shown in Fig. 1. Thus the absolute value of $\left\{\hat{s}^{\prime}(m)\right\}$ is calculated in such an averaged manner as follows:

$$
\overline{\left|s_{i}^{\prime}\right|}=\frac{1}{M} \sum_{m=0}^{M-1}\left|\hat{s}_{i}^{\prime}(m)\right|
$$

Moreover, the frequency of the phases $\left\{L \hat{s}_{i}^{\prime}(m)\right\}$ is plotted on the circumference as shown in Fig. 1 and w.r.t. the horizontal axis from 0 to 360 [deg] as shown in Fig. 2. In these figures, as indicated by the dashed line, the direction (phase) in which the frequency takes a peak value corresponds with the direction (phase) passing through the center of a cluster. Thus the phase where the frequency becomes maximum is denoted as $\angle s_{i-p e a k}^{\prime}$ and is adopted as the estimate of $\angle s_{i}^{\prime}$. The solid line in the figures implies the phase $\angle s_{i}$ of the transmitted symbol $s_{i}$.

By substitution of $\overline{\left|s_{i}^{\prime}\right|}$ and $\angle s_{i-p e a k}^{\prime}$ into Eq. (16), the absolute value and the phase of $h_{i}$ are estimated as $\left|\hat{h}_{i}\right|=\overline{\left|s_{i}^{\prime}\right|} /\left|s_{i}\right|$ and $\angle \hat{h}_{i}=\angle s_{i-\text { peak }}^{\prime}-\angle s_{i}$, respectively. Therefore, the estimate of the fading coefficients can be finally obtained as follows:

$$
\hat{h}_{i}=\left|\hat{h}_{i}\right| e^{j L \hat{h}_{i}}
$$

The constellations of $\left\{s_{i}(m)\right\}$ and $\left\{s_{i}^{\prime}(m)\right\}$ in the case of 16QAM are shown in Fig. 3. As found from this figure, there exist two types of direction (phase) in the constellation of the transmitted symbols' $\left\{s_{i}(m)\right\}$ : one is the direction passing through two points and the other is the direction passing through only a single point. Accordingly there exist two types of direction (phase) in the constellation of the provisional symbols $\left\{s_{i}^{\prime}(m)\right\}$ : one is the direction passing through the centers of two clusters as indicated by the dot-dash line and the other is the direction passing through the center of a single cluster as indicated by the dashed line. Furthermore, the frequency of $\left\{\angle s_{i}^{\prime}(m)\right\}$ is plotted along the horizontal axis from 0 to 360 [deg] as shown in Fig. 


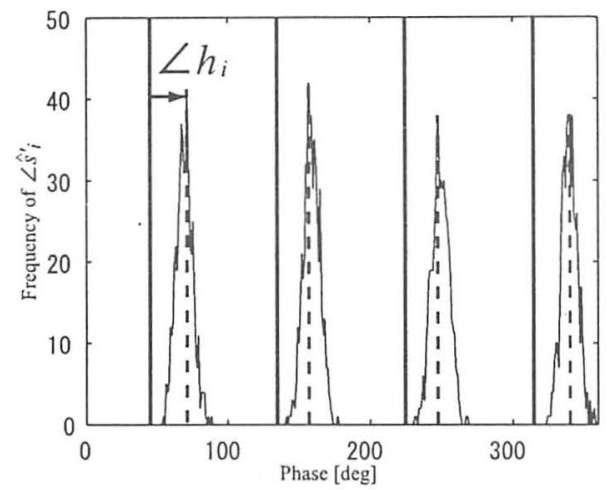

Fig. 2: Frequency distribution of $\angle \hat{s}_{i}^{\prime}$ (4QAM).

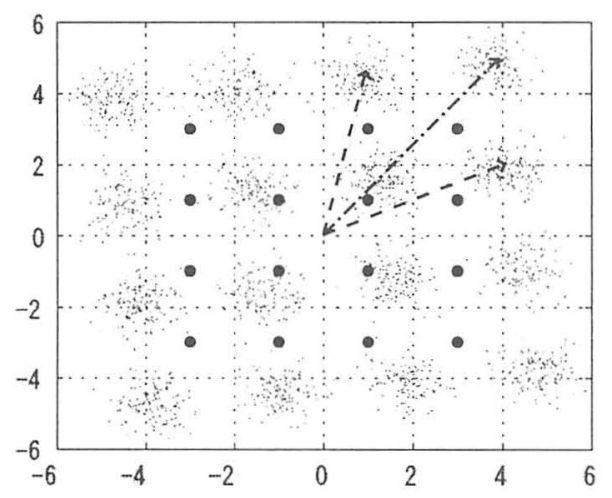

Fig. 3: Constellation of $s_{i}$ and $\hat{s}_{i}^{\prime}$ (16QAM).

4. Then it is found from the figure that the frequency takes a peak for the direction (phase) passing through the centers of two clusters and is twice as large as the frequency for the direction passing through the center of a single cluster.

Therefore we can also estimate the fading coefficients in 16QAM by calculating the averaged absolute value $\overline{\left|s_{i}^{\prime}\right|}$ and searching the phase $\angle s_{i-\text { peak }}^{\prime}$ of the restored provisional symbols and then by comparing them with the averaged absolute value $\left|s_{i}\right|(=\sqrt{2}+\sqrt{10} / 2)$ and the 16 known phases $\angle s_{i}$ of the transmitted symbols. The fading coefficient estimation above is also valid in the case of 64QAM and 256QAM when the signal to noise ratio is high.

\subsection{Restoration of transmitted symbols}

In order to clarify the relation between the abovementioned provisional symbol, the channel estimation and the symbol restoration, Eq. (14) is rewritten as $\hat{s}^{\prime}(m)=W x(m)$ in terms of the de-mixing matrix $W=\hat{A}^{-1}$ resulting from the algorithm in 2.4 . In 3.2 , according to this equation, the provisional symbols $\left\{\hat{s}^{\prime}(m)\right\}$ are restored from the received OFDM symbols $\{\boldsymbol{x}(m)\}$. At every channel, the fading coefficient $\hat{h}_{i}$ is estimated, based on the frequency of these restored provisional symbols $\left\{\hat{s}_{i}^{\prime}(m)\right\}$. For the same restored

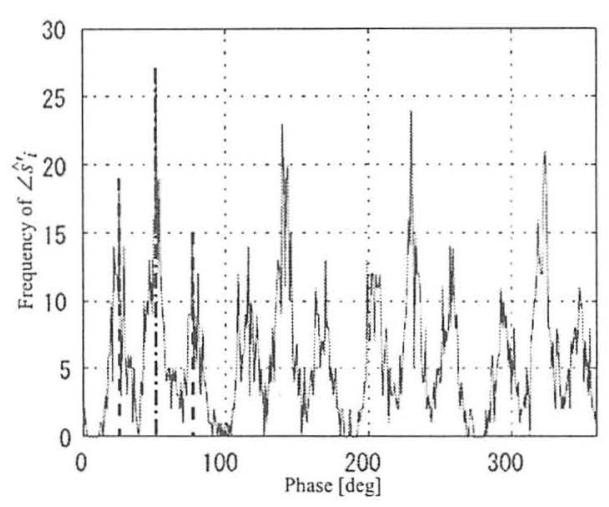

Fig. 4: Frequency distribution of $\angle \hat{s}_{i}^{\prime}$ (16QAM).

provisional symbols, the synchronous detection is made using $\hat{h}_{i}$ to restore the transmitted symbols as follows:

$$
\hat{s}_{i}(m)=\hat{s}_{i}^{\prime}(m) / \hat{h}_{i} \quad(i=0,1, \cdots, N-1)
$$

As seen from the above discussion, at least two buffers are needed for storing the $M$ recieved symbols in our proposed method. Therefore application of the proposed method is difficult for the real-time processing under the time-variant channels because of large delays due to the buffering and the processing for CFO and channel estimation.

\section{Simulations}

To verify the effectiveness of the proposed method, several simulations were carried out for 16QAMOFDM with $N=50$ sub-carriers using the gray code. Concretely the transmitted symbols $\left\{s_{i}(m) \mid i=\right.$ $0,1, \cdots, N-1 ; m=0,1, \cdots, M-1\}$ with $M=2,048$ were generated by uniform random number, and the received symbols $\left\{x_{l}(m) \mid l=0,1, \cdots, N-1 ; m=\right.$ $0,1, \cdots, M-1\}$ were given as follows:

$$
x_{l}=\sum_{i=0}^{N-1} \alpha_{l i} h_{i} s_{i}+n_{l}
$$

where $h_{i}$ denotes the fading coefficient at the $i$-th subcarrier, $\alpha_{l i}$ denotes the interference intensity from $i$-th sub-carrier to $l$-th sub-carrier, and $n_{l}$ denotes Gaussian white noise with zero mean and with the variance determined inversely from the signal-to-noise (SN) ratio defined as follows:

$$
\mathrm{SNR}=10 \log _{10} \frac{E\left[\left|\sum_{i=0}^{N-1} \alpha_{l i} h_{i} s_{i}\right|^{2}\right]}{E\left[\left|n_{l}\right|^{2}\right]}[\mathrm{dB}]
$$

The fading coefficients $\left\{h_{i}\right\}$ were determined so that the phase should be within $\pm \pi / 4$ as follows. At first, the Rayleigh fading model was created by use of the frequency correlation characteristics between sub-carriers 
[13] when the ratio of the delay spread to OFDM symbol length was set to 0.01. The complex number resulting from the Rayleigh fading model was denoted as $\tilde{h}_{i}$. Next, a positive real value $c$ was determined so that the phase of $h_{i}=\tilde{h}_{i}+c$ should be within $\pm \pi / 4$ for all subcarriers. The complex number $h_{i}$ obtained in this manner was adopted as the fading coefficient at the $i$ th subcarrier. Furthermore, the nonlinear function in Eq. (4) was given as $\phi(u)=|u|^{2} \operatorname{sgn}(u)$ [9], the update coefficient was set as $\eta=0.0001$, and the initial value of the uninterference matrix was given as $W_{0}=I$.

A hundred Monte Carlo trials were made on each of the combination of CFO $\epsilon(=0,0.05, \cdots, 0.5)$ and $\operatorname{SNR}(=15,20,25[\mathrm{~dB}])$. In each trial, the uninterference matrix $W$ was updated until 50 epochs: the standard NG algorithm was used in the first 15 epochs and in the second 35 epochs the conditional NG algorithm [7]. The estimation accuracy for CFO was evaluated by mean square errors $\left(\mathrm{MSE}=\sum_{K=1}^{100}\left|\hat{\epsilon}_{K}-\epsilon\right|^{2} / 100\right)$ and the estimation accuracy for symbols was evaluated by Bit Error Rate (BER).

\subsection{Estimation accuracy}

The estimated results of $\mathrm{CFO}$ are shown in Fig. 5. From this figure, it is found that CFO is accurately estimated since MSE takes the values near $10^{-4} \sim 10^{-5}$ in $\epsilon \leq 0.45$. Fig. 6 shows the BER results for the received symbol $x$ (w/o Compensation), the estimated provisional symbol $\hat{s}^{\prime}$ (CFO Cancellation) and the restored symbol $\hat{s}$ (Proposed Method). From the figure it is seen that BER for $x$ decreases as CFO increases but BER for $\hat{s}^{\prime}$ keeps constant while both of them are not dependent on the $\mathrm{SN}$ ratios. Although BER is better for $\hat{s}^{\prime}$ than for $x$, the fact that BER for $\hat{s}^{\prime}$ is 0.2 means that $\hat{s}^{\prime}$ is badly subjected to the channel distortion.

On the other hand, in the range of $\epsilon=0 \sim 0.4$, BER for $\hat{s}$ takes the values under $0.07,0.01,0.001$, at SNR $=15,20,25[\mathrm{~dB}]$, respectively. Moreover, BER for $\hat{s}$ is improved about 3, 20, 200 times as much as BER for $\hat{s}^{\prime}$. It was also confirmed that BER obtained by use of the true values of the flat fading coefficients is almost coincident with BER for $\hat{s}$. From these results it is judged that the fading compensation by the proposed method is valid.

Although the algorithm in 2.4 progresses so that the independency between each element of the separated symbol $u$ should be maximized, the algorithm has a possibility that the overlearning occurs since the algorithm is not provided with the convergence criterion. For this reason, the values of MSE and BER are considered smaller at $\epsilon=0.35$ than at $\epsilon=0$. The fact that the variation of $\mathrm{BER}$ is not smooth is also considered due to the effect of the overlearning.

\subsection{Estimation results of fading coefficients}

The accuracy of the estimated fading coefficient $\hat{h}_{i}$ is evaluated by the gain $\left|\hat{h}_{i}\right|$ and the phase $\angle \hat{h}_{i}$. Figure 7 shows the estimation results of $\hat{h}_{i}$ when CFO is $\epsilon=$

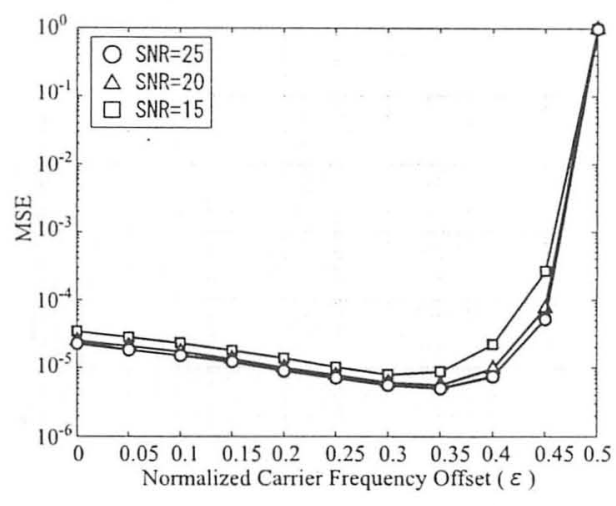

Fig. 5: Mean square errors at $\mathrm{CFO} \epsilon=0$ to 0.5 .

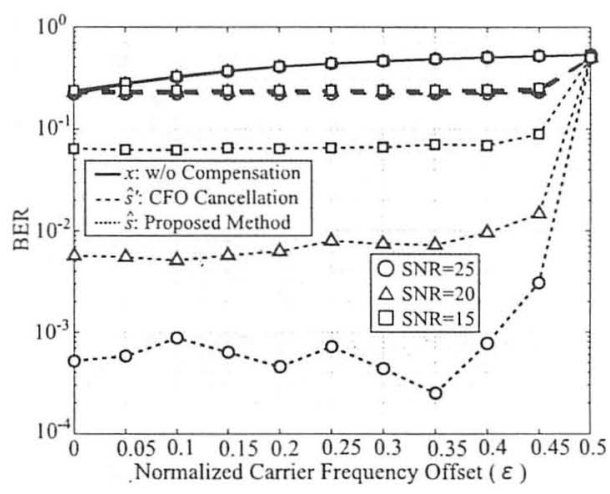

Fig. 6: BER performance at $\mathrm{CFO} \epsilon=0$ to 0.5 .

0.3 and SNR is $25[\mathrm{~dB}]$ : The mean and the standard deviation of the gain estimation results $\left|\hat{h}_{i}\right|$ in addition to the true gain are plotted in Fig. $7(\mathrm{a})$, and those of the phase estimation results $\angle \hat{h}_{i}$ in addition to the true phase in Fig. 7(b). From the results of Fig. 7 (a) and (b), it is found that the gain $\left|h_{i}\right|$ and the phase $\angle h_{i}$ are estimated quite well at every sub-carrier.

By comparison of the results at $\mathrm{SNR}=15,20,25[\mathrm{~dB}]$, it was found that the estimation accuracy (standard deviation) of $\left|\hat{h}_{i}\right|$ and $L \hat{h}_{i}$ becomes worse as SNR increases, of which tendency is stronger in the phase estimation. This is because, effected by the additive noise $n_{l}$, the frequency distribution (Fig. 4) of $\angle \hat{s}_{i}^{\prime}$ becomes so dull that peak search for estimation of $\angle h_{i}$ becomes difficult around angles $\angle h_{i} \simeq \pm \pi / 4$. On the other hand, any large differences in the accuracy of $\mathrm{CFO}$ estimation could not be discerned in the range of $\mathrm{CFO}$ from 0 to 0.4 .

From these results, the fading coefficient estimation by the proposed method is considered valid under CFO environments while SNR is high. 


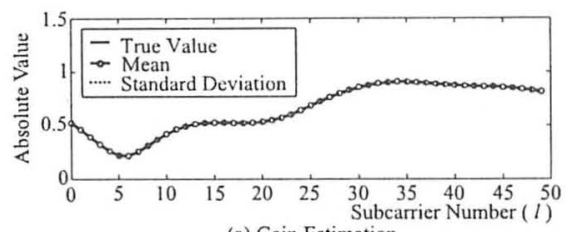

(a) Gain Estimation

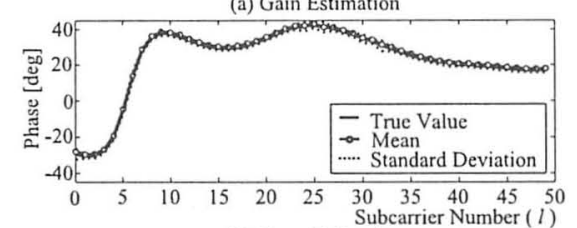

(b) Phase Estimation

Fig. 7: Absolute Value and phase estimation of $h_{i}(\epsilon=$ $0.3, \mathrm{SNR}=25[\mathrm{~dB}])$.

\section{Conclusions}

In this paper, based on NG algorithm, we have proposed the blind CFO and channel estimation using only received symbols without any pilot symbols. Based on the these estimation results, we have explained how to restore the unknown transmitted symbols. It has been also confirmed from the results of simulation carried out for 16QAM-OFDM systems that the proposed method is valid and that the CFO and the fading coefficients are well estimated when SNR is relatively high.

Application of the proposed method can be expected in the field of space radio communication where Doppler shift is inevitable to cause CFO. The reasons are as follows. In the space communication between a satellite and an earth-station or between satellites, it takes from several ten minutes to several hours for the space search satellite (the sender) to deliver its observation data such as planet pictures to the earth-station (the receiver) through radio wave, e.g., it takes about 80 minutes from Saturn to Earth. In such a case, the data are generally transmitted or received while a line-ofsight channel is kept between the transmitter and the receiver. Thus it is required to restore the observation data from transiently received signals but it is difficult to request the sender to transmit the same observational data agairr. In such a line-of-sight channel, it is considered that the influence due to reflective waves is little and thus the phase fluctuation of the fading coefficients is in less range than $\pm \pi / 4$. These are the reasons why application of our approach can be expected in the field of space radio communication.

\section{References}

[1] M. Itami, "Fundamentals of OFDM and Its Application," Fundamentals Review, Vol.1, No. 2, pp.35-43, Oct. 2007. (in Japanese).
[2] L. Tong, B.M. Sadler, M. Dong, "Pilot-assisted wireless transmissions," IEEE Signal Processing Magazine, vol. 21, no. 6, pp.12-25, Nov. 2004.

[3] J.J. van de Beek, M. Sandell, and P.O. Börjesson, "ML estimation of time and frequecy offset in OFDM systems," IEEE Trans. Signal Processing., vol.45, no.7, pp.1800-1805, July. 1997.

[4] T. Keller, L. Piazzo, P. Mandarini, and L. Hanzo, "Orthogonal frequency division multiplex synchronization techniques for frequency-selective fading channels," IEEE Journal on Selected Areas in Communications, vol.19, no.6, pp.999-1008, June, 2001.

[5] M. Ghogho, and A. Swami, "Carrier frequency synchronization for OFDM systems," in Handbook of Signal Processing for Mobile Communications Handbook, ed.M. Ibnkahla, Ch.8, CRC Press, 2004.

[6] B. Chen, H. Wang, "Blind estimation of OFDM carrier frequency offset via oversampling," IEEE Trans. Signal Processing., vol.52, no.7, pp.20472057, July. 2004.

[7] F. Nakagawa, S. Takase, H. Shiratsuchi, H. Gotanda, "Estimation of OFDM Carrier Frequency Offset and Symbol Recovery by ICA," IEICE Trans. Fundamentals, Vol. J91-A, No. 04, pp.448-457, Apr. 2008. (in Japanese).

[8] H. Sakai, K. Hayashi, "Block Signal Transmission and Frequency Domain Equalization," IEICE Trans. Fundamentals, Vol. 87, No.10, pp.855-860, Oct. 2004. (in Japanese).

[9] S. Amari, S. C. Douglas, A. Cichoki, and H. H. Yang, "Multichannel blind deconvolution and equalization using the natural gradient," Proc. on Signal Processing Advance in Wireless Communication Workshop, pp.101-104, Paris, France, 1997.

[10] P. Smaragdis, "Blind separation of convolved mixtures in the frequency domain," Neurocomputing, vol.22, pp.21-34,1998

[11] N. Murata, S. Ikeda, and A. Ziehe, "An approach to blind source separation based on temporal structure of speech signals," Neurocomputing, vol.41, no.1-4, pp.1-24, 2001.

[12] H. Gotanda et al., "Permutation correction and speech extraction based on split spectrum through FastICA," Proc.ICA2003, pp.379-384, 2003.

[13] Jean-Paul M.G. Linnartz, "Multi-Carrier Channel Simulation," JPL's Wireless Communication Referen-ce Website

(http://wireless.per.nl/reference/chaptr05/ofdm/ ofdmchan.htm), 1995. 